\title{
Alimentação de Hoplias aff. malabaricus (Bloch, 1794) e Oligosarcus robustus Menezes, 1969 em uma lagoa sob influência estuarina, Pelotas, RS
}

\author{
Fabiano Corrêa ${ }^{1 *}$ \\ Sérgio Renato Noguez Piedras ${ }^{2}$ \\ ${ }^{1} \mathrm{PPG}$ Lato sensu em Ecologia Aquática Costeira, Instituto de Oceanografia \\ Laboratório de Ictiologia, Universidade Federal do Rio Grande \\ Caixa Postal 474, CEP 96201-900, Rio Grande - RS, Brasil \\ ${ }^{2}$ Laboratório de Ictiologia, Departamento de Zootecnia, FAEM \\ Universidade Federal de Pelotas, Caixa Postal 354, CEP 9610-610, Pelotas - RS, Brasil \\ *Autor para correspondência \\ correafecologia@yahoo.com.br
}

Submetido em 02/02/2009

Aceito para publicação em 26/05/2009

\section{Resumo}

Alimentação de Hoplias aff. malabaricus (Bloch, 1794) e Oligosarcus robustus Menezes, 1969 em uma lagoa sob influência estuarina, Pelotas, RS. O objetivo do presente trabalho foi o de estudar a dieta de Hoplias aff. malabaricus e Oligosarcus robustos na Lagoa Pequena, com o intuito de avaliar a importância de organismos estuarinos na alimentação dessas espécies. Foram identificados 12 itens alimentares, sendo peixes o recurso mais frequente na dieta de ambas as espécies. Peixes eurialinos como a tainha Mugil platanus e o peixe-rei Odontesthes argentinensis foram itens comuns na dieta das duas espécies, caracterizando um comportamento oportunista por incluir na sua alimentação não apenas peixes de água doce, mas também peixes oriundos do ambiente estuarino adjacente, integrando as cadeias alimentares estuarinas e de água doce.

Unitermos: dieta, peixe, carnívoro, estuário, Lagoa Pequena

\section{Abstract}

Feeding of Hoplias aff. malabaricus (Bloch, 1794) and Oligosarcus robustus Menezes, 1969 in a lagoon under estuarine influence, Pelotas, RS. The objective of the present paper was to study the diets of Hoplias aff. malabaricus and Oligosarcus robust in "Pequena Lagoon" and evaluate the importance of estuarine organisms in the feeding of freshwater fish. A total of twelve food categories were identified and it was established that the fish resource was the most frequent in the diet of both species. Euryhaline fish such as Mugil platanus mullets and Odontesthes argentinensis silversides were common items in the diets of these two species, which included not only freshwater fish but also euryhaline fish from the adjacent estuarine zone, integrating both resources in the food chain.

Key words: diet, fish, carnivore, estuary, Pequena Lagoon 


\section{Introdução}

A plasticidade trófica é o termo utilizado para descrever a flexibilidade dos animais em utilizar recursos alimentares disponíveis no ambiente. Trata-se de uma característica marcante da ictiofauna tropical sendo especialmente importante em ambientes cuja disponibilidade é variável (Abelha et al., 2001), como por exemplo em zonas estuarinas (Chaves e Umbria, 2003). Nessas áreas, a composição faunística e consequentemente a disponibilidade de alimento, variam de acordo com as condições hidrodinâmicas.

Para as espécies de água doce que vivem em ambientes submetidos à influência estuarina a capacidade de utilizar os recursos alimentares provenientes do estuário pode ser crucial para o seu sucesso neste tipo de ambiente. De fato, Garcia et al. (2001) mostraram que na Lagoa Pequena, ambiente submetido à influencia físico-química e biológica da zona estuarina da Lagoa dos Patos, sul do Brasil, a movimentação de peixes entre o ambiente estuarino e límnico pode ser uma importante via de subsídio lateral de energia conectando as tramas tróficas estuarinas e de água doce.

Hoplias aff. malabaricus e Oligosarcus robustus são exemplos de espécies dulcícolas que ocorrem na Lagoa Pequena. Hoplias aff. malabaricus (Bloch, 1794), conhecida popularmente como traíra, é um peixe neotropical pertencente à família Erythrinidae, que possui ampla distribuição geográfica, ocorrendo em todas as bacias hidrográficas da América do Sul, com exceção da área transandina e dos rios da Patagônia (Fowler, 1950; Nelson, 1994). Oligosarcus robustus pertence à família Characidae, tendo distribuição no sistema da Lagoa dos Patos e no sistema lagunar costeiro do sul do Brasil, Uruguai e Argentina (Koch et al., 2000). Apesar de existirem diversos estudos sobre dieta destas espécies (Loureiro e Hahn, 1996; Hahn et al., 1997; Carvalho et al., 2002; Nunes e Hartz, 2006), o conhecimento da influência de áreas estuarinas no perfil trófico ainda são incipientes.

Ambas as espécies, provavelmente, apresentam adaptações para lidar com as condições ambientais encontradas na Lagoa Pequena, notadamente a influencia físico-quimica e biológica do estuário. Parte destas adaptações pode estar relacionada com a capacidade dessas espécies em utilizar recursos alimentares provenientes do estuário. Assim, para testar esta hipótese, este estudo objetivou descrever a dieta de H. malabaricus e O. robustus na Lagoa Pequena, sul do Brasil.

\section{Materiais e Métodos}

A Lagoa Pequena apresenta uma superfície aproximada de 4.700 hectares localizada entre os paralelos $31^{\circ} 33^{\prime}$ e $33^{\circ} 38^{\prime} \mathrm{S}$ e $52^{\circ} 02^{\prime}$ e $52^{\circ} 06^{\prime} \mathrm{W}$ (Figura 1), e inunda terras dos municípios de Pelotas e Turuçu. Possui pelo lado noroeste uma ligação com o arroio Corrientes e seus afluentes menores, e a sudeste o canal Corrientes, a principal comunicação com a Lagoa dos Patos o que torna a Lagoa Pequena parte integrante do sistema estuarial e diferenciando-a de um corpo essencialmente límnico (Jica/SCPp-RS, 2000). Devido sua proximidade com a Colônia de pescadores Z-3 de Pelotas, a Lagoa Pequena é usada como local de pesca, nos períodos em defeso das espécies estuarinas, quando a captura é direcionada para as espécies de água doce.

As amostragens foram realizadas durante junho, julho, agosto e setembro de 2005. Para as coletas dos indivíduos utilizou-se redes de espera com malhas de 20,30, 40 e $50 \mathrm{~mm}$ entre-nós, sendo expostas por $24 \mathrm{~h}$ e com despesca a cada $12 \mathrm{~h}$. Após a captura, os espécimes foram acondicionados em caixa isotérmica com gelo, e transferidos ao Laboratório de Limnologia da Universidade Católica de Pelotas, onde se obteve os seguintes dados biométricos; comprimento total em $(\mathrm{mm})$ e peso total em (g). Posteriormente, os estômagos foram retirados e fixados em formol a $10 \%$, para posterior análise do conteúdo estomacal.

Foi determinado, para cada item alimentar, a frequência de ocorrência ( $\mathrm{Fo} \%)$, o qual corresponde à frequência percentual do número de estômagos em que foi registrado o recurso alimentar em relação ao número de estômagos com alimento e peso relativo (Pi\%), pelo qual o peso é expresso percentualmente em relação ao peso de todos os recursos alimentares presentes no estômago (Hyslop, 1980). Os itens alimentares foram pesados em balança analítica e identificados com auxílio de bibliografia especializada até a menor categoria taxonômica possível. 


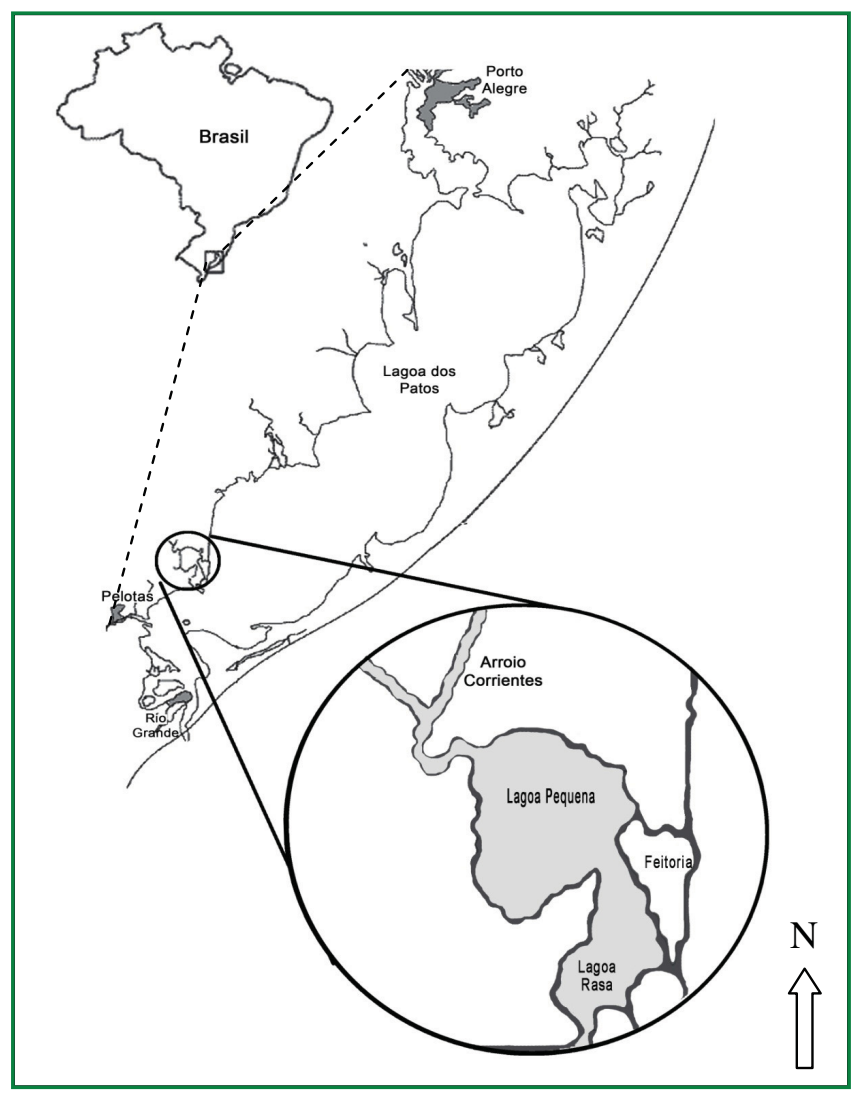

FIGURA 1: Sistema da Lagoa dos Patos com a localização da Lagoa Pequena $\left(31^{\circ} 33^{\prime}\right.$ a $31^{\circ} 38^{\prime} \mathrm{S}$ e $52^{\circ} 02^{\prime}$ a $\left.52^{\circ} 06^{\prime} \mathrm{W}\right)$, na região sul da Lagoa dos Patos.

Os dados de frequência de ocorrência e peso relativo de cada recurso alimentar foram combinados no Índice Alimentar (IAi) de acordo com (Kawakami e Vazzoler, 1980), seguindo a fórmula: $\mathrm{Fo} \% \times \mathrm{Pi} \% / \sum \mathrm{Fo} \% \times \mathrm{Pi} \%$; Onde: $\mathrm{Fo}=$ frequência de ocorrência do item $\mathrm{i} ; \mathrm{Pi}=$ peso relativo do item i. Esse índice alimentar permite distinguir mais adequadamente a importância relativa de cada item (Kawakami e Vazzoler, 1980). Adicionalmente para cada indivíduo das espécies estudadas foi verificado o grau de repleção gástrica (Andrian et al., 1994), de acordo com as seguintes categorias: estômago vazio; estômago parcialmente vazio; estômago parcialmente cheio e estômago cheio.

A mensuração das variáveis físico-químicas da água foram feitas no local das amostragens, a $20 \mathrm{~cm}$ da superfície. A temperatura do ar e da água e oxigênio dissolvido foram verificados utilizando oxímetro marca YSI modelo 55; a condutividade por condutivímetro marca Corning modelo CD-55; pH por potenciômetro marca Instrutherm modelo RTS-28. A alcalinidade, matéria orgânica e gás carbônico foram determinados em laboratório por volumetria seguindo a metodologia de (APHA, 1998).

\section{Resultados e Discussão}

Os parâmetros físico-químicos encontram-se dentro dos padrões esperados (Esteves, 1998) (Tabela 1), com exceção da elevada condutividade e salinidade obtida. Isso provavelmente deve-se, a presença de água salobra proveniente do estuário da Lagoa dos Patos.

TABELA 1: Descritores físico-químicos do local de amostragem na Lagoa Pequena, Pelotas, RS.

\begin{tabular}{lc}
\hline Parâmetros físico-químicos & Valores \\
\hline Temperatura do ar & $16,8^{\circ} \mathrm{C}$ \\
Temperatura da água & $18,3^{\circ} \mathrm{C}$ \\
$\mathrm{pH}$ & 7,0 \\
$\mathrm{CO}_{2}$ & $2,5 \mathrm{mg} / \mathrm{L}^{-1}$ \\
$\mathrm{O}_{2}$ & $8,8 \mathrm{mg} / \mathrm{L}^{-1}$ \\
Alcalinidade & $11,0 \mathrm{mg} / \mathrm{L}^{-1}$ \\
Condutividade & $7,400 \mu \mathrm{mols} / \mathrm{cm}$ \\
Salinidade & $10,0 \mathrm{~g} / \mathrm{L}$ \\
\hline
\end{tabular}

Foram analisados 96 indivíduos, 66 de Hoplias aff. malabaricus e 30 de Oligosarcus robustus. Dos 66 espécimes de $H$. aff. malabaricus 42 eram fêmeas e 24 machos, cujo comprimento total médio foi de $320 \pm 39 \mathrm{~mm}$ e peso total médio de $414,1 \pm 188,9 \mathrm{~g}$. Segundo o grau de repleção gástrica de Hoplias aff. malabaricus apenas $16,7 \%$ dos indivíduos apresentaram o estômago cheio, $10,6 \%$ parcialmente cheio, $15,1 \%$ parcialmente vazio e a maioria 57,6\% apresentou o estômago vazio. Entre os 30 indivíduos capturados da espécie $O$. robustus todos eram fêmeas, os quais apresentaram comprimento total médio de $290 \pm 23 \mathrm{~mm}$ e peso total médio de $250,9 \pm 66,7 \mathrm{~g}$. Destes $16,7 \%$ apresentaram o estômago cheio, 43,3\% estômago parcialmente cheio, $0 \%$ parcialmente vazio e $40 \%$ estômago vazio.

De maneira geral, as espécies apresentaram padrões de repleção gástrica semelhantes no que se refere à proporção de indivíduos com estômagos cheios e na alta proporção de indivíduos com estômagos vazios. 
Estes resultados são compatíveis com os obtidos por Barbieri et al. (1982), que encontraram maiores frequências de estômagos vazios para exemplares de $H$. aff. malabaricus na represa do Lobo nos meses frios. De fato, a alta proporção de estômagos vazios obtida neste estudo deve estar associada à época do ano (inverno), pois a temperatura de $16,8^{\circ} \mathrm{C}$ pode ter influenciado na atividade alimentar das espécies. Bistoni et al. (1995) relatam que o número de estômagos sem alimento em peixes carnívoros e/ou piscívoros é comum em estações frias. De acordo com Menezes (1969), a captura de presas pode ter uma elevação durante mudanças entre estações frias para estações quentes, o qual favorece a atividade de forrageamento. Além disso, espécies que consomem peixes como $H$. aff. malabaricus e $O$. robustus são capazes de passar por longos períodos em jejum. Paiva (1974) relata que a espécie $H$. aff. malabaricus na fase adulta alimenta-se pouco devido aos hábitos sedentários permanecendo longos períodos sem obtenção de alimento.

Foi observada uma diversidade de 12 itens alimentares, sendo nove consumidos por $H$. aff. malabaricus e oito por O. robustus (Tabela 2). Das nove categorias alimentares que compuseram a dieta de $H$. aff. malabaricus, o tambicu Oligosarcus jenynsii foi à presa que obteve maior valor no índice alimentar ( $\mathrm{IAi}=0,36)$, seguido do peixe-rei Odontesthes argentinensis com ( $\mathrm{IAi}=0,22)$, a tainha Mugil platanus com (IAi $=0,17)$, resto de peixe ( $\mathrm{IAi}=0,15)$, o biru Cyphocharax voga com (IAi=0,04), o cará Geophagus brasiliensis (IAI $=0,03)$ e o restante variando entre 0,02 a 0,003, considerando Hemíptera como um item ocasional por demonstrar um baixo valor no IAi. Já para $O$. robustus, o item mais importante foi peixe-rei (Odontesthes argentinensis) $(\mathrm{IAi}=0,37)$ seguido do tambicu (Oligosarcus jenynsii) (IAi $=0,28)$. Os demais itens variaram entre $\mathrm{IAi}=0,123 \mathrm{a}<0,001$.

Embora o crustáceo Farfantepenaeus paulensis não fosse registrado na alimentação de $H$. aff. malabaricus, vale destacar que Gurgel et al. (2005), estudando a alimentação da comunidade de peixes de um trecho do rio Ceará, Estado do Rio Grande do Norte, observaram como principais itens alimentares dessa espécie crustáceos $(\mathrm{IAi}=0,57)$ seguido de peixes $(\mathrm{IAi}=0,32)$ e insetos (IAi 0,10). A pequena contribuição de crustáceos na dieta de $H$. aff. malabaricus pode estar relacionado à menor disponibilidade deste recurso no inverno, período em que este estudo foi realizado, já que o camarão e outros crustáceos decápodes tornam-se abundantes no estuário da Lagoa dos Patos (Bemvenuti, 1998), principalmente na primavera e verão, época em que estão na fase de crescimento e alimentação.

TABELA 2: Índice dos recursos consumidos por Hoplias aff. malabaricus e Oligosarcus robustus durante o inverno na Lagoa Pequena, Pelotas - RS

\begin{tabular}{|c|c|c|}
\hline \multirow{2}{*}{ Recursos alimentares } & \multicolumn{2}{|l|}{ IAi } \\
\hline & H. aff. malabaricus & O. robustus \\
\hline Algas filamentosas & & 0,06 \\
\hline \multicolumn{3}{|l|}{ Crustacea } \\
\hline $\begin{array}{l}\text { Farfantepenaeus pau- } \\
\text { lensis }\end{array}$ & & 0,04 \\
\hline \multicolumn{3}{|l|}{ Insecta } \\
\hline Coleoptera & & 0,03 \\
\hline Hemiptera & 0,003 & \\
\hline \multicolumn{3}{|l|}{ Mollusca } \\
\hline Gastropoda & & $<0,001$ \\
\hline \multicolumn{3}{|l|}{ Peixes de água doce } \\
\hline \multicolumn{3}{|l|}{ Characidae } \\
\hline $\begin{array}{l}\text { Astyanax fasciatus } \\
\text { (Cuvier,1819) }\end{array}$ & 0,02 & \\
\hline $\begin{array}{l}\text { Oligosarcus jenynsii } \\
\text { (Gunther, 1864) }\end{array}$ & 0,36 & 0,28 \\
\hline \multicolumn{3}{|l|}{ Chiclidae } \\
\hline $\begin{array}{l}\text { Geophagus brasiliensis } \\
\text { (Quoy \& Gaimard, 1824) }\end{array}$ & 0,03 & \\
\hline \multicolumn{3}{|l|}{ Curimatidae } \\
\hline $\begin{array}{l}\text { Cyphocharax voga } \\
\text { (Hensel, 1870) }\end{array}$ & 0,04 & \\
\hline \multicolumn{3}{|l|}{ Peixes de água salgada } \\
\hline \multicolumn{3}{|l|}{ Atherinidae } \\
\hline $\begin{array}{l}\text { Odontesthes argentinen- } \\
\text { sis (Valenciennes, 1835) }\end{array}$ & 0,22 & 0,37 \\
\hline \multicolumn{3}{|l|}{ Mugilidae } \\
\hline $\begin{array}{l}\text { Mugil platanus (Gun- } \\
\text { ther, 1880) }\end{array}$ & 0,17 & 0,09 \\
\hline \multicolumn{3}{|l|}{ Outros } \\
\hline $\begin{array}{l}\text { Resto de peixe (tecido } \\
\text { mucular, barbatana e } \\
\text { escama) }\end{array}$ & 0,15 & 0,12 \\
\hline $\begin{array}{l}\text { Alimento não identifi- } \\
\text { cado }\end{array}$ & 0,02 & \\
\hline
\end{tabular}

Ficou evidente a influência da entrada de água 
mixohalina na Lagoa Pequena na alimentação de $H$. aff. malabaricus como em O. robustus, uma vez que, espécies de peixes típicos de ambiente estuarino, como a tainha Mugil platanus e o peixe-rei Odontesthes argentinensis, estiveram presentes nas análises do conteúdo estomacal (Figura 2). Para $H$. aff. malabaricus $15,4 \%$ do conteúdo alimentar foi constituído por Mugil platanus e Odontesthes argentinensis (Figura 3). Já a dieta de O. robustus $18.7 \%$ foi constituída de Farfantepenaeus paulensis, Odontesthes argentinensis e Mugil platanus, todos de origem estuarina (Figura 3). $\mathrm{O}$ consumo destes itens por $H$. aff. malabaricus e $O$. robustus reforçam a importância dos estuários, não só como áreas de criação, mas também como ambientes provedores de recursos para espécies predadoras de água doce. Esses resultados vem de encontro com a constatação de Leung e Camargo (2005), que relatam que espécies de peixes marinho-estuarinas têm importante influência na alimentação de peixes de água doce.

Os peixes foram itens alimentares mais representativos na dieta de ambas as espécies. Dos itens identificados apenas $O$. argentinensis, $M$. platanus e restos de peixe foram semelhantes para as duas espécies. A predominância de peixes na dieta de Hoplias aff. malabaricus tem sido descrita por diversos autores em diferentes ecossistemas (Gulgel e Canan, 1999; Carvalho et al., 2002; Cassemiro et al., 2005), entre outros. De maneira geral, contribuição de outros itens como fragmentos vegetais e insetos são poucos significativos (Loureiro e Hahn, 1996). Para Oligosarcus robustus os peixes foram identificados como principal item alimentar, caracterizando a espécie como carnívoro oportunista. Resultados semelhantes foram obtidos por Nunes e Hartz (2006), que registraram peixes, insetos e crustáceos na dieta de $O$. robustus na Lagoa Fortaleza, Rio Grande do Sul, classificando a espécie como piscívora. Hartz et al. (1996) encontraram para $O$. jenynsii na lagoa do Caconde/RS, uma dieta baseada principalmente em peixes e camarões, destacando a similaridade entre os itens ingeridos por espécies do gênero Oligosarcus.

Cazorla et al. (2003) descrevem Oligosarcus jenynsii como insetívoro no rio Sauce Grande na província de Buenos Aires, enquanto que para SilvaHermes et al. (2004), O. jenynsii na região do alto rio Uruguai, é uma espécie carnívora com propensão a piscívoria. Barros (2004) classifica $O$. jenynsii, do rio Juramento na Argentina como uma espécie generalista com predominância de peixes, camarões e larvas de insetos, sendo considerado um peixe macro-carnívoro

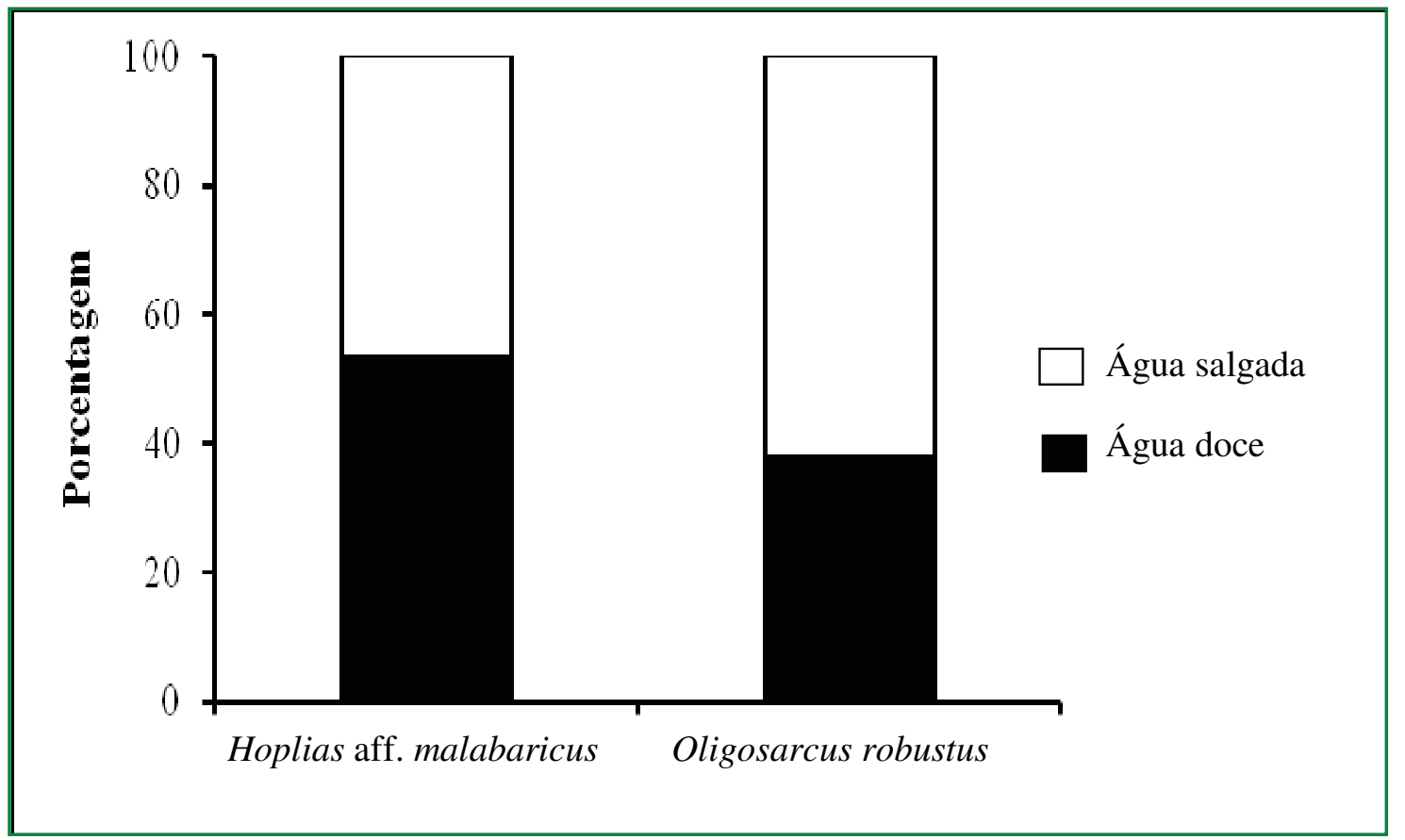

FIGURA 2: Porcentagem do indice alimentar considerando os peixes consumidos por Hoplias aff. malabaricus e Oligosarcus robustus em função da sua origem. 


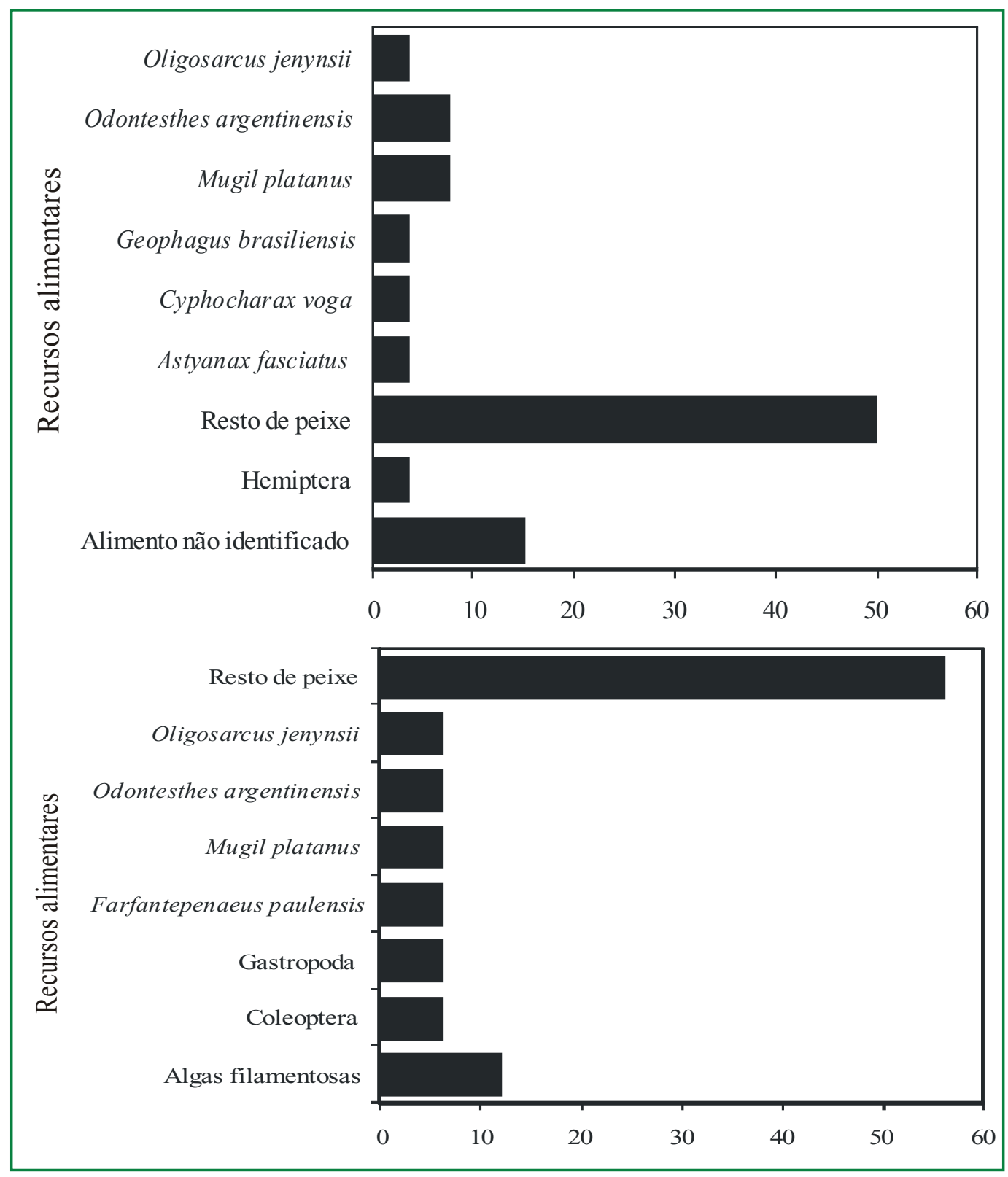

FIGURA 3: Frequência de ocorrência dos itens alimentares consumidos por Hoplias aff. malabaricus (acima) e Oligosarcus robustus (abaixo) durante o inverno na Lagoa Pequena, Pelotas, RS.

com tendência a ictiofagia em tamanhos maiores. Esse fato pode ser aplicado à espécie O. robustus, pois apresentou uma dieta semelhante à $O$. jenynsii, consumindo peixe, camarão e inseto este ultimo tendo uma baixa ocorrência. A baixa importância das algas filamentosas na dieta de O. robustus sugere ingestão acidental. É provável que as algas sejam oriundas do sistema digestivo da tainha, já que algas são itens comuns em sua dieta.
Estudos complementares poderão verificar como a dieta destas espécies é influenciada pelas alterações sazonais na Lagoa Pequena. Apesar do presente estudo não ter contemplado a sazonalidade, os resultados obtidos se destacam por registrar a ocorrência de peixes estuarino-dependentes, como o peixe-rei Odontesthes argentinensis e a tainha Mugil platanus, na alimentação de Hoplias aff. malabaricus e Oligosarcus robustus, reforçando o oportunismo destas espécies e a importância 
da elevada produção secundária estuarina como um fator de sustentação para as populações de peixes que habitam os corpos de águas dulcícolas no entorno do estuário da Lagoa dos Patos.

\section{Agradecimentos}

Ao laboratorista Nilton Jair da Silva e ao funcionário Paulo Jesus Freitas de Oliveira pela amizade e auxilio nas coletas realizadas durante o estudo.

\section{Referências}

Abelha, M. C. F.; Agostinho, A. A.; Goulart, E. 2001. Plasticidade trófica em peixes de água doce. Acta Scientiarum, 23 (2): 425 434.

Alvim, A. C. C. 1999. Composição e alimentação da ictiofauna em um trecho do alto rio São Francisco, município de Três Marias, Minas Gerais. Dissertação de Mestrado, Universidade Federal de São Carlos, Brasil, 97pp.

APHA - American Public Health Association. 1998. Standard methods for the examination of water and wastewater. APHA, New York, USA, 824pp.

Andrian, I. F.; Dória, C. R. C.; Torrente, G. 1994. Espectro alimentar e similaridade na composição da dieta de quatro espécies de Leporinus (Characiformes. Anostomidae) do rio Paraná, Brasil. Revista Unimar, 16 (3): 97-106.

Barbieri, G.; Verani, J. R.; Barbieri, M. C. 1982. Dinâmica quantitativa da nutrição de Hoplias malabaricus (Bloch, 1794) na represa do Lobo (Brotas-Itirapina-SP) (Pisces, Erythrinidae). Revista Brasileira de Biologia, 42 (2): 295-302.

Barros, E. S. 2004. Alimentación de Oligosarcus jenynsii (Characiformes: Characidae) em dos embales sobre el rio Juramento. Salta. Subtrópico de Argentina. Revista AquaTIC, 20: 44-50.

Bemvenuti, C. E. 1998. Invertebrados bentônicos. In: Seeliger, U.; Odebrecht, C. \& Castello, J. P. (Eds). Os ecossistemas costeiros e marinhos do extremo sul do Brasil. $1^{\text {a }}$ ed. ECOSCIENTIA, Rio de Janeiro, Brasil, p.46-51.

Bistoni, M. de los A.; Haro, J. G.; Gutiérrez, M. 1995. Feeding of Hoplias malabaricus in the wetlands of Dulce River (Cordoba, Argentina). Hydrobiology, 316: 103-107.

Carvalho, N. L.; Fernandes, C. H. V.; Moreira, V. E. S. 2002. Alimentação de Hoplias malabaricus (Bloch, 1794) (Osteichtyes, Erythrinidae) no rio Vermelho, Pantanal Sul Mato-Grossense. Revista de Brasileira de Zoociências, 4 (2): 227-236.

Cazorla, L. A.; Durán, W.; Tejera, L. 2003. Alimentación de la ictiofauna del río Sauce Grande, provincia de Buenos aires, Argentina. Biologia Acuática, 20: 73-79.

Cassemiro, F. A. S.; Hahn, N. S.; Delariva, R. L. 2005. Estrutura trófica da ictiofauna, ao longo do gradiente longitudinal do reservatório de Salto Caxias (rio Iguaçu, Paraná, Brasil) no terceiro ano após o represamento. Acta Scientiarum, 27 (1): 67-71.
Chaves, P. T. C.; Úmbria, S. C. 2003. Changes in the composition of transitry fishes in coastal systems, estuary and continental shelf. Brazilian Archives of Biology and Technology, 46 (1): 41-46.

Esteves, F. A. 1998. Fundamentos de Limnologia. $2^{\text {a }}$ ed. Interciência, Rio de Janeiro, Brasil, 602pp.

Fowler, H. W. 1950. Os peixes da água doce do Brasil. Arquivos de Zoologia do Estado de São Paulo, 6 (2): 205-404.

Garcia, A. M.; Vieira, J. P.; Winemiller, K. O. 2001. Dynamics of the shallow-water fish assemblage of the Patos Lagoon estuary (Brazil) during cold and warm ENSO episodes. Journal of Fish Biology, 59: 1218-1238.

Gurgel, H. C. B.; Canan, B. 1999. Feeding of six species in Jiqui lagoon eastern coast of Rio Grande do Norte, Brazil. Acta Scientiarum, 21 (2): 243-246.

Gurgel, H. C. B.; Silva, N. B.; Lucas, F. D.; Souza, L. L. G. 2005. Alimentação da comunidade de peixes de um trecho do rio Ceará Mirim, em Umari, Taipu, Estado do Rio Grande do Norte, Brasil. Acta Scientiarum, 27 (2): 229-233.

Hahn, N. S.; Andrian, I. de F.; Fugi, R.; Almeida, V. L. L. 1997. Ecologia trófica. In: Hartz, S. M.; Martins, A. \& Barbieri, G. (Eds). Dinâmica da alimentação e dieta de Oligosarcus jenynsii (Gunter, 1864) na Lagoa do Caconde, Rio Grande do Sul, Brasil (Teleostei, Characidae). Boletim do Instituto de Pesca, 23 (único): 21-29.

Hyslop, E. J. 1980. Stomach contents analysis: A review of methods and their application. Journal Fish Biology, 17: 411-429.

Jica/SCPp-RS. 2000. The study on the environmental management of the hydrographic Brazil of Patos and Mirin Lakes in the Federative Republic of Brazil. Final Report. 4v. Kokusai Kogyo/Pacific Consultants International, Tokyo, Japão, 38pp.

Kawakami, E.; Vazzoler, G. 1980. Método gráfico e estimativa de índice alimentar aplicado no estudo de alimentação de peixes. Boletim Instituto Oceanográfico, 29 (2): 250-207.

Koch, W. R.; Milani, P. C.; Grosser, K. M. 2000. Guia ilustrado de peixes do Parque do Delta do Jacuí. $1^{\text {a }}$ ed. Fundação Zoobotânica do Rio Grande do Sul/DOLIKA, Porto Alegre, Brasil, 91pp.

Leung, R.; Camargo, A. F. M. 2005. Marine influence on fish assemblage in coastal streams of Itanhaém river basin, southeastern Brazil. Acta Limnológica Brasiliensia, 17 (2): 219-232.

Loureiro, V. E.; Hahn, N. S. 1996. Dieta e atividade alimentar da traíra, Hoplias malabaricus (Bloch, 1794) (Osteichtyes, Erythrinidae), nos primeiros anos de formação do reservatório de Segredo-Paraná. Acta Limnológica Brasiliensia, 8: 195-205.

Menezes, N. A. 1969. The food of Brycon and three closely related genera of the tribe Acestrorhynchini. Papeis Avulsos Zoologia, 22 (20): 271-223.

Nelson, J. S. 1994. Fishes of the world. $3^{\text {rd }}$ ed. John Wiley is Sons, New York, USA, 600pp.

Nobriga, M. L.; Feyer, F. 2008. Diet composition in San Francisco Estuary striped bass: Does trophic adaptability have its limits? Environmental Biology Fish, 83: 495-503.

Nunes, D. M.; Hartz, S. M. 2006. Feeding dynamics and ecomorphology of Oligosarcus jenynsii (Gunter, 1864) and Oligosarcus robustus (Menezes, 1969) in the lagoa Fortaleza, Southern Brazil. Brazil. Journal of Biology, 66 (1): 121-132. 
Paiva, M. P. 1974. Crescimento, reprodução e alimentação da traíra, Hoplias malabaricus (Bloch, 1794), no nordeste brasileiro, Fortaleza. Tese de Doutorado. Universidade Federal do Ceará, Brasil, 32pp.

Silva-Hermes. S.; Meurer, S.; Filho, Z. E. 2004. Biologia alimentar e reprodução do peixe-cachorro (Oligosarcus jenynsii Gunter, 1864) na região do alto rio Uruguai-Brasil. Acta Scientiarum, 26 (2): 175-179.

Zavala-Camin, L. A. 1996. Introdução aos estudos sobre alimentação natural em peixes. EDUEM, Maringá, Brasil, $129 \mathrm{pp}$. 